удК 330.43

Н. В. Мамонова

Байкальский государственный университет, г. Иркутск, Российская Федерация

\title{
ИССЛЕДОВАНИЕ РЫНКА НЕДВИЖИМОСТИ В ГОРОДЕ ШЕЛЕХОВЕ
}

\begin{abstract}
АНнотАЦия. В статье рассматривается анализ рынка жилья на примере моногорода Шелехов за последние два года. Целью исследования является выявление факторов, влияющих на формирование цен на недвижимость в моногородах России. Объектом исследования выступил вторичный рынок жилья данного города, а именно, были рассмотрены однокомнатные квартиры. Однокомнатные квартиры были выбраны как самый популярный вариант приобретения недвижимости для семей с разными доходами. В результате исследования были выделены некоторые факторы, которые наиболее ощутимо влияют на повышение цены жилой недвижимости: общая площадь квартиры, наличие застекленного балкона, этажность дома и площадь кухни. Результаты исследования могут быть полезны при прогнозировании тенденций развития рынка недвижимости в моногородах, для выбора оптимальной стратегии при оценке объектов недвижимости или для целей налогообложения.

кЛЮчЕВЫЕ СЛОВА. Анализ цен; рынок недвижимости; формирование стоимости; эконометрический анализ.

ИНФОРМАЦИЯ О СТАТЬЕ. Дата поступления 19 февраля 2017 г.; дата принятия к печати 2 марта 2017 г.; дата онлайн-размещения 31 марта 2017 г.

\section{N. V. Mamonova Baikal State University, Irkutsk, Russian Federation}

\section{INVESTIGATION OF REAL ESTATE MARKET IN THE SHELEKHOV TOWN}

\begin{abstract}
The article considers analysis of the housing market in terms of the Shelekhov monotown during last two years. The goal of research is identification of the factors that influence the real estate price formation in Russia's monotowns. The object of investigation is the secondary housing market in this town, that is, consideration of one-room flats. The one-room flats are chosen as the most popular variant of acquiring real estate by families with various incomes. The result of the investigation is identification of some factors that most tangibly influence the increase of housing real estate price: the total living space, the availability of a glassed-in balcony, the number of stories in the building, and the kitchen space. The results of the investigation may appear useful in forecasting the trends of real estate market development in monotowns, in selecting optimal strategy in estimating real estate objects or inn taxation purposes. KEYWORDS. Analysis of prices; real estate market; cost formation; econometric analysis.
\end{abstract}

ARTICLE INFO. Received February 19, 2017; accepted March 2, 2017; available online March 31, 2017.

В современном мире проблема любого эконометрического исследования российской экономики становится очень актуальной. Исследование, связанное с прогнозом, ведет к важным результатам. Поэтому очень актуальны различные эконометрические методики расчетов той или иной экономической ситуации, например, связанные с рынком недвижимости.

Рынок недвижимости - это сектор национальной рыночной экономики, который связывает в себе различные процессы производства - от создания жилья, потребления до управления рынком жилья.

(C) Н. В. Мамонова, 2017

\section{Baikal Research Journal}


В результате исследования были рассмотрены 136 объявлений по продаже однокомнатных квартир на вторичном рынке жилья города Шелехов Иркутской области за последние два года ${ }^{1}$. Покупатели чаще всего обращали внимание на следующие важные факторы.

Стоимость квартиры существенно зависела от ее жилой площади, а также площади кухни. С увеличением площади растет и цена квартиры. Эти факторы необходимо выделить отдельно как самостоятельные регрессоры.

Помимо общей площади есть еще и такой оцениваемый факт, как удобство расположения всех помещений, высота потолков и изношенность жилого фонда. Поэтому одним из главных факторов становится номер серии построенного дома и его год эксплуатации. Этажность квартиры также определяет наличие в доме лифта и мусоропровода, которые зачастую не всегда работают, что опять-таки сказывается на цене.

При сборе информации обнаружилось, что продавцом часто указывается наличие телефона, железной двери и домофона. Телефон обеспечивает удобства жильцу, также наличие телефонной точки определяет возможность подключения к сети Интернет. Домофон и железная дверь обеспечивают безопасность квартиры и подъезда.

И, конечно, немаловажный фактор, который было решено включить в модель, это место расположение продаваемой квартиры. С одной стороны, для людей близость к центру - это значимый фактор для определения надежности транспортной связи с практически любой точкой города, с другой стороны, центр дает возможность разнообразия удовлетворения культурных потребностей.

Данное исследование было проведено с помощью регрессионного анализа [1-4], где зависимая переменная $y$ - цена стоимости однокомнатной квартиры (в тыс. p.) зависела от нескольких факторов-регрессоров:

$-x_{1}$ - жилая площадь квартиры, $\mathrm{m}^{2}$;

$-x_{2}$-площадь кухни, $\mathrm{m}^{2}$;

и несколько качественных факторов, имеющих только два возможных значения: 0 и 1 :

$-x_{3}-$ серия и год построенного дома: $1-$ если год новой постройки, $0-$ если дома старой серии;

$-x_{4}-$ этажность дома: 1 - если квартира расположена не на первом и не на последнем этажах; 0 - в противном случае;

$-x_{5}$ - наличие телефона, домофона и железной двери;

- $x_{6}$ - удаленность от центра: 1 - удаленность существенная, 0 - допустима и удовлетворяет покупателей.

Были построены различные линейные и нелинейные модели [5; 6], и получены следующие результаты.

Оцененная линейная модель множественной регрессии:

$$
\widehat{y}_{i}=50,51+0,75 x_{1}+1,8 x_{2}+3,39 x_{3}+1,76 x_{4}+5,82 x_{5}+0,2 x_{6},
$$

здесь коэффициенты при переменных $x_{i}$ означают возможное изменение в среднем цены за квартиру на соответствующее значение. Например, если жилую площадь квартиры $x_{1}$ увеличить на $1 \mathrm{~m}^{2}$, то цена однокомнатной квартиры возрастет в среднем на 0,75 тыс. р.

Качество построенной модели описывают коэффициентом детерминации $R^{2}$. В нашем исследовании $R^{2}=0,73$, что позволяет сделать вывод о том, что вариация рассматриваемых факторов за последние два года в г. Шелехов на 73 \% объясняет вариацию ее цены, а оставшиеся 27 \% приходятся на другие неучтенные в модели факторы, например, платежеспособность покупателя.

\footnotetext{
${ }^{1}$ Недвижимость Иркутска и Шелехова [Электронный ресурс]. URL: http://www.dom-irk.ru.
}

\section{Baikal Research Journal}


Статистическую значимость модели в целом проверили дисперсионным анализом в регрессии [7]. Наблюдаемое значение критерия Фишера $F_{\text {факт }}=7,81$, в сравнении с критической точкой $F_{\kappa p}=(0,05 ; 6,17)$, на $5 \%$ уровне значимости сви-

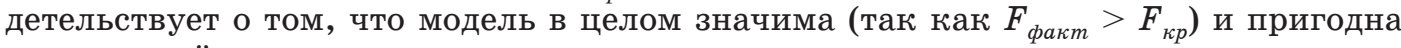
для дальнейшего прогноза.

Проведем углубленный корреляционный анализ рассматриваемых факторов, для того чтобы выявить наиболее информативный. Известно, что мерой линейной связи между двумя величинами служит коэффициент корреляции $r_{i j}[8$, с. 60]. Подсчитаем коэффициенты парной корреляции между любыми двумя факторами $x_{i}$ и $x_{j}$ по формуле:

$$
r_{B}=\hat{r}=\frac{n \Sigma\left(x_{i} \cdot x_{j}\right)-\Sigma x_{i} \cdot \Sigma x_{j}}{\sqrt{\left[n \Sigma\left(x_{i}\right)^{2}-\left(\Sigma x_{i}\right)^{2}\right] \cdot\left[n \Sigma\left(x_{j}\right)^{2}-\left(\sum x_{j}\right)^{2}\right]}} .
$$

Для расчета данных коэффициентов использовался пакет прикладных программ Excel [9]. Была получена матрица парных коэффициентов корреляции и представлена в табл. 1.

Таблица 1

Матрица парной корреляиии факторов

\begin{tabular}{|r|r|r|r|r|r|r|r|}
\hline & \multicolumn{1}{|c|}{$x_{1}$} & \multicolumn{1}{c|}{$x_{2}$} & \multicolumn{1}{c|}{$x_{3}$} & \multicolumn{1}{c|}{$x_{4}$} & \multicolumn{1}{c|}{$x_{5}$} & \multicolumn{1}{c|}{$x_{6}$} & \multicolumn{1}{c|}{$x_{7}$} \\
\hline$x_{1}$ & 1 & & & & & & \\
\hline$x_{2}$ & 0,0617 & 1 & & & & & \\
\hline$x_{3}$ & 0,1806 & 0,7438 & 1 & & & & \\
\hline$x_{4}$ & 0,1324 & 0,7671 & 0,8718 & 1 & & & \\
\hline$x_{5}$ & 0,1276 & 0,4097 & 0,7406 & 0,5535 & 1 & & \\
\hline$x_{6}$ & 0,0710 & 0,0441 & 0,2115 & 0,1848 & 0,2447 & 1 & \\
\hline$x_{7}$ & $-0,3614$ & $-0,1585$ & $-0,1850$ & $-0,1993$ & $-0,0750$ & 0,0389 & 1 \\
\hline
\end{tabular}

Проанализировав матрицу парной корреляции можно сделать вывод, что между третьим и четвертым факторами существует высокая корреляция. Значит факторы общая площадь и жилая площадь квартиры взаимосвязаны, так как $\left|r_{i j}\right|<0,8$. А наименьшая корреляционная связь присутствует между $x_{6}$ и $x_{7}$. Следовательно, мы показали, что этаж квартиры не зависит от материала дома.

Оценим значимость полученных коэффициентов корреляции разными способами, чтобы выявить наиболее информативный фактор, для этого проверим основную гипотезу о не значимости данного коэффициента $H_{0}: r=0$ при конкурирующей гипотезе $H_{1}: r \neq 0$ различными способами.

1 способ. Проверим основную гипотезу с помощью построения доверительных интервалов [2, с. 58]. Доверительный интервал - это интервал, который с надежностью $\gamma$, близкой к $100 \%$, содержит истинное неизвестное значение параметра. В нашем исследовании зададим надежность исследования, равной $95 \%$. Доверительные интервалы строятся с помощью $z$-преобразования Фишера

$$
z^{\prime}-t_{\gamma} \frac{1}{\sqrt{n-l-3}}<r_{i j}<z^{\prime}+t_{\gamma} \frac{1}{\sqrt{n-l-3}},
$$

где величину $z^{\prime}$ находим из таблицы $z$-преобразования Фишера, $t_{\gamma}-$ критическое значение Стьюдента, $n-$ количество наблюдений, $l$ - порядок коэффициента корреляции.

Используя эту же таблицу, находим верхнюю и нижнюю границы для коэффициентов корреляции. Если построенный доверительный интервал не содержит нулевое значение, то основную гипотезу следует отклонить, и считать, что меж-

\section{Baikal Research Journal}


ду рассматриваемыми факторами существует значимая корреляционная зависимость. Получим следующие оценки коэффициентов корреляции (табл. 2).

Интервальные оценки для коэффициентов корреляции

\begin{tabular}{|c|c|c|c|c|c|c|}
\hline & $x_{1}$ & $x_{2}$ & $x_{3}$ & $x_{4}$ & $x_{5}$ & $x_{6}$ \\
\hline$x_{1}$ & $-0,1<r_{12}<0,23$ & $0,01<r_{13}<0,34$ & $-0,03<r_{14}<0,3$ & $-0,04<r_{15}<0,29$ & $-0,1<r_{16}<0,24$ & $-0,5<r_{17}<-0,2$ \\
\hline$x_{2}$ & & $0,66<r_{23}<0,81$ & $0,68<r_{24}<0,82$ & $0,25<r_{25}<0,53$ & $-0,12<r_{26}<0,2$ & $-0,31<r_{27}<0,02$ \\
\hline$x_{3}$ & & & $0,82<r_{24}<0,91$ & $0,66<r_{35}<0,81$ & $0,04<r_{36}<0,37$ & $-0,34<r_{37}<-0,01$ \\
\hline$x_{4}$ & & & & $0,42<r_{45}<0,66$ & $0,01<r_{46}<0,34$ & $-0,35<r_{47}<-0,02$ \\
\hline$x_{5}$ & & & & & $0,07<r_{56}<0,39$ & $-0,24<r_{57}<0,1$ \\
\hline$x_{6}$ & & & & & & $-0,14<r_{67}<0,2$ \\
\hline
\end{tabular}

Из полученной таблицы можно сделать вывод, что основная гипотеза принимается для коэффициентов $r_{12}, r_{14}, r_{15}, r_{16}, r_{26}, r_{27}, r_{57}, r_{67}$.

2 способ. Используем критерий Стьюдента ( $t$-критерий) [10] для проверки основной гипотезы

$$
t_{\text {факт }}=\frac{r_{B} \sqrt{n-l-2}}{\sqrt{1-r_{B}^{2}}} \sim t(n-l-2),
$$

где $l$ - порядок коэффициента корреляции, т. е. число фиксируемых при его оценке переменных. Для коэффициентов парной корреляции $l=0$.

В результате получаем наблюдаемые значения критерия (табл. 3).

Таблица 3

Значения $t_{\text {факт }}$ для коэффициентов парной корреляции

\begin{tabular}{|r|r|r|r|r|r|r|}
\hline & $x_{2}$ & $x_{3}$ & \multicolumn{1}{c|}{$x_{4}$} & \multicolumn{1}{c}{$x_{5}$} & \multicolumn{1}{c|}{$x_{6}$} & \multicolumn{1}{c|}{$x_{7}$} \\
\hline$x_{1}$ & 0,7156 & 2,1252 & 1,5463 & 1,4887 & 0,8237 & $-4,4874$ \\
\hline$x_{2}$ & & 12,8833 & 13,8435 & 5,1986 & 0,5116 & $-1,8578$ \\
\hline$x_{3}$ & & & 20,5985 & 12,7572 & 2,5049 & $-2,1796$ \\
\hline$x_{4}$ & & & & 7,6925 & 2,1768 & $-2,3542$ \\
\hline$x_{5}$ & & & & & 2,9213 & $-0,8708$ \\
\hline$x_{6}$ & & & & & & 0,4510 \\
\hline
\end{tabular}

Критическое значение критерия

$$
t_{\kappa p}=t\left(1-\frac{\alpha}{2}, n-l-2\right)
$$

найдено по специальной таблице [8, с. 60] при уровне значимости $\alpha=0,05$

$$
t_{\kappa p}=t(0,975 ; 134)=1,978 .
$$

Из табл. 3 видно, что большинство значений $\left|t_{\text {факт }}\right|<t_{\kappa p}$, следовательно, основную гипотезу $H_{0}: r=0$ необходимо отвергнуть, таким образом, на $5 \%$ уровне значимости можно сделать вывод, что практически все рассматриваемые коэффициенты корреляции значимы, кроме коэффициентов $r_{12}, r_{14}, r_{15}, r_{16}, r_{26}$, $r_{27}, r_{57}, r_{67}$.

В результате проведенного исследования между общей и жилой площадью существует очень тесная корреляционная зависимость (с повышением общей площади увеличивается и жилая площадь). Самый низкий коэффициент между вторым и шестым факторами (он равен 0,044), т. е. между количеством комнат и этажом (см. табл. 1). Поэтому, в дальнейшем исследовании этими показателями можно пренебречь.

\section{Baikal Research Journal}


3 способ. Проверим основную гипотезу, используя таблицу Фишера-Иейтса ${ }^{2}$ [2, с. 371]. Выбираем из специальной таблицы по заданному уровню значимости $\alpha=0,05$ и числу степеней свободы $d f=n-1-2=134$, где $n-$ число наблюдений $(n=136)$ и $l-$ порядок коэффициента корреляции $(l=0)$, критическую точку, $r_{\kappa p} \approx 0,18$. Сравнивая полученные коэффициенты $\left|r_{i j}\right|$ с критическим значением критерия, основную гипотезу $H_{0}$ для значений, которые превышают критическую отметку, следует отвергнуть.

Проведем сравнительный анализ наблюдаемых точек из табл. 1 (табл. 4)

Сравнительная матрица парной корреляции факторов

Таблица 4

\begin{tabular}{|r|r|r|r|r|r|r|r|}
\hline & \multicolumn{1}{|c|}{$x_{1}$} & \multicolumn{1}{|c|}{$x_{2}$} & \multicolumn{1}{c|}{$x_{3}$} & \multicolumn{1}{c|}{$x_{4}$} & \multicolumn{1}{c|}{$x_{5}$} & \multicolumn{1}{c|}{$x_{6}$} & \multicolumn{1}{c|}{$x_{7}$} \\
\hline$x_{1}$ & 1 & & & & & & \\
\hline$x_{2}$ & 0,0617 & 1 & & & & & \\
\hline$x_{3}$ & $\mathbf{0 , 1 8 0 6}$ & $\mathbf{0 , 7 4 3 8}$ & 1 & & & & \\
\hline$x_{4}$ & 0,1324 & $\mathbf{0 , 7 6 7 1}$ & $\mathbf{0 , 8 7 1 8}$ & 1 & & & \\
\hline$x_{5}$ & 0,1276 & $\mathbf{0 , 4 0 9 7}$ & $\mathbf{0 , 7 4 0 6}$ & $\mathbf{0 , 5 5 3 5}$ & 1 & & \\
\hline$x_{6}$ & 0,0710 & 0,0441 & $\mathbf{0 , 2 1 1 5}$ & $\mathbf{0 , 1 8 4 8}$ & $\mathbf{0 , 2 4 4 7}$ & 1 & \\
\hline$x_{7}$ & $-\mathbf{0 , 3 6 1 4}$ & $-0,1585$ & $-\mathbf{0 , 1 8 5 0}$ & $-\mathbf{0 , 1 9 9 3}$ & $-0,0750$ & 0,0389 & 1 \\
\hline
\end{tabular}

Выделенные элементы (полученные ранее коэффициенты) очевидно больше критического значения, для которых гипотеза о не значимости отвергается, т. е. коэффициенты корреляции - значимы. В результате данного исследования, получаем те же элементы матрицы парной корреляции незначимыми, что и во втором методе, и еще элемент $r_{12}$.

В результате проведенных исследований по всем трем методам можно сделать вывод, что почти все коэффициенты корреляции, описывающие линейную зависимость между рассматриваемыми факторами, являются значимыми, кроме $r_{14}$, $r_{15}, r_{16}, r_{26}, r_{27}, r_{57}, r_{67}$. Однако на рассматриваемые коэффициенты корреляции могут влиять и другие факторы, которые также участвуют в модели, поэтому для более углубленного анализа воспользуемся исследованием частных коэффициентов корреляции $r_{i j / x}$.

Теперь найдем частные коэффициенты корреляции, которые характеризуют тесноту связи между двумя факторами при устранении влияния остальных рассматриваемых факторов. Они вычисляются по формуле

$$
r_{i j / \ldots}=-\frac{q_{i j}}{\sqrt{q_{i i} \cdot q_{j j}}},
$$

где $q_{i j}$ - алгебраическое дополнение матрицы коэффициентов парной корреляции. Полученные результаты сведем в табл. 5 .

Таблица 5

Частные коэффициенты корреляиии

\begin{tabular}{|r|r|r|r|r|r|r|r|}
\hline & \multicolumn{1}{|c|}{$x_{1}$} & \multicolumn{1}{|c|}{$x_{2}$} & \multicolumn{1}{c|}{$x_{3}$} & \multicolumn{1}{c|}{$x_{4}$} & \multicolumn{1}{c|}{$x_{5}$} & \multicolumn{1}{c|}{$x_{6}$} & \multicolumn{1}{c|}{$x_{7}$} \\
\hline$x_{1}$ & 1 & $-0,0998$ & 0,1314 & $-0,0488$ & $-0,0298$ & 0,0506 & $-0,3473$ \\
\hline$x_{2}$ & $-0,0998$ & 1 & 0,3436 & 0,3043 & $-0,2209$ & $-0,1545$ & $-0,0096$ \\
\hline$x_{3}$ & 0,1314 & 0,3436 & 1 & 0,6284 & 0,6494 & 0,0445 & $-0,0200$ \\
\hline$x_{4}$ & $-0,0488$ & 0,3043 & 0,6284 & 1 & $-0,1905$ & 0,0938 & $-0,0764$ \\
\hline$x_{5}$ & $-0,0298$ & $-0,2209$ & 0,6494 & $-0,1905$ & 1 & 0,0958 & 0,0541 \\
\hline$x_{6}$ & 0,0506 & $-0,1545$ & 0,0445 & 0,0938 & 0,0958 & 1 & 0,0881 \\
\hline$x_{7}$ & $-0,3473$ & $-0,0096$ & $-0,0200$ & $-0,0764$ & 0,0541 & 0,0881 & 1 \\
\hline
\end{tabular}

${ }^{2}$ URL: http://www.studfiles.ru/preview/1720882/.

\section{Baikal Research Journal}


В данной таблице коэффициенты $r_{i j / x}$ означают, что рассматривается связь между факторами $x_{i}$ и $x_{i}$, исключая все остальные факторы. Самый высокий коэффициент частной корреляции между третьим и четвертым факторами, исключая все остальные, что совпадает с результатами, полученными из анализа коэффициентов парной корреляции факторов. А самая слабая связь прослеживается между факторами $x_{3}$ - общая площадь и $x_{7}$ - материал дома.

Оценим значимость данных коэффициентов частной корреляции аналогичным образом, что и коэффициенты парной корреляции. Проверим основную гипотезу о незначимости данных коэффициентов $H_{0}: r=0$ при конкурирующей гипотезе $H_{1}: r \neq 0$ разными способами.

1 способ. Проверим основную гипотезу с помощью построения доверительных интервалов [2, с. 58]. Исследование проводится с надежностью $95 \%$. При построении доверительных интервалов справедлива формула:

$$
z^{\prime}-t_{\gamma} \frac{1}{\sqrt{n-l-3}}<r_{i j / x}<z^{\prime}+t_{\gamma} \frac{1}{\sqrt{n-l-3}},
$$

где величину $z^{\prime}$ находим из таблицы $z$-преобразования Фишера. Используя эту же таблицу, находим верхнюю и нижнюю границы для частных коэффициентов корреляции. Если построенная интервальная оценка содержит в себе нулевое значение, то основную гипотезу $H_{0}$ следует принять. Широкий доверительный интервал указывает на то, что оценка неточна, узкий - на точную оценку. Для коэффициентов частной корреляции из табл. 5 получаем доверительные интервалы, представленные в табл. 6.

Интервальные оченки для частных коэффициентов корреляции

\begin{tabular}{|c|c|c|c|c|c|c|}
\hline & $x_{2}$ & $x_{3}$ & $x_{4}$ & $x_{5}$ & $x_{6}$ & $x_{7}$ \\
\hline$x_{1}-0,26<r_{12 \mid \ldots}<0,08$ & $-0,04<r_{13 \mid \ldots}<0,3$ & $-0,21<r_{14 \mid \ldots}<0,13$ & $-0,19<r_{15 \mid \ldots . .}<0,15$ & $-0,12<r_{16 \mid \ldots}<0,22$ & $-0,49<r_{17 \mid \ldots}<-0,17$ \\
\hline$x_{2}$ & & $0,17<r_{23 \mid \ldots}<0,48$ & $0,17<r_{24 \mid \ldots}<0,45$ & $-0,38<r_{25 \mid \ldots}<-0,05$ & $-0,31<r_{26 \mid \ldots}<0,02$ & $-0,17<r_{27 \mid \ldots}<0,17$ \\
\hline$x_{3}$ & & & $0,51<r_{34 \mid \ldots}<0,96$ & $0,53<r_{35 \mid \ldots}<0,74$ & $-0,13<r_{36 \mid \ldots}<0,21$ & $-0,19<r_{37 \mid \ldots}<0,15$ \\
\hline$x_{4}$ & & & & $-0,35<r_{45 \mid \ldots}<-0,02$ & $-0,08<r_{46 \mid \ldots}<0,26$ & $-0,24<r_{47 \mid \ldots}<0,1$ \\
\hline$x_{5}$ & & & & & $-0,08<r_{56 \mid \ldots}<0,26$ & $-0,12<r_{57 \mid \ldots}<0,22$ \\
\hline$x_{6}$ & & & & & & $-0,09<r_{67 \mid \ldots}<0,25$ \\
\hline
\end{tabular}

В результате основную гипотезу $H_{0}$ отвергаем для коэффициентов $r_{23 / \ldots}, r_{24 / \ldots}$, $r_{34 / \ldots}, r_{25 / \ldots}, r_{35 / \ldots}, r_{45 / \ldots}, r_{17 / \ldots}$, а все остальные коэффициенты незначимы, так как доверительный интервал содержит нулевое значение.

2 способ. Для анализа частных коэффициентов корреляции применим способ с использованием $t$-критерия Стьюдента [10]. Рассчитаем наблюдаемые значения критерия для каждого частного коэффициента корреляции из табл. 5

$$
t_{\text {факт }}=\frac{r_{B} \sqrt{n-l-2}}{\sqrt{1-r_{B}^{2}}} \sim t(n-l-2),
$$

где $l$ - порядок коэффициента корреляции, т. е. число фиксируемых при его оценке переменных, a $n$ - число наблюдений (количество квартир, участвующих в анализе). Полученные результаты приведены в табл. 7.

Из таблицы можно сделать вывод, что при уровне значимости $\alpha=0,05$ частные коэффициенты корреляции $r_{23 / \ldots}, r_{24 / \ldots}, r_{34 / \ldots}, r_{25 / \ldots}, r_{35 / \ldots}, r_{45 / \ldots}, r_{17 / \ldots}$ значимы, так как для них $\left|t_{\text {कакт }}\right|>t_{\text {кр }}$, при критическом значении критерия Стьюдента $t_{\kappa p}=t(0,975 ; 129)=1,979$. В таком случае основную гипотезу $H_{0}: r=0$ о не значимости коэффициентов частной корреляции следует отвергнуть. Остальные

\section{Baikal Research Journal}


коэффициенты не значимы. Полученные результаты совпадают с результатами, полученными первым способом.

Значения $t_{\text {факт }}$ для частных коэффициентов корреляции

\begin{tabular}{|r|r|r|r|r|r|r|}
\hline & $x_{2}$ & \multicolumn{1}{|c|}{$x_{3}$} & \multicolumn{1}{|c|}{$x_{4}$} & \multicolumn{1}{c|}{$x_{5}$} & \multicolumn{1}{c|}{$x_{6}$} & \multicolumn{1}{c|}{$x_{7}$} \\
\hline$x_{1}$ & $-1,1389$ & 1,5054 & $-0,5544$ & $-0,3384$ & 0,5751 & $-4,2067$ \\
\hline$x_{2}$ & & 4,1561 & 3,6276 & $-2,5721$ & $-1,7761$ & $-0,1090$ \\
\hline$x_{3}$ & & & 9,1752 & 9,7002 & 0,5058 & $-0,2273$ \\
\hline$x_{4}$ & & & & $-2,2045$ & 1,0703 & $-0,8703$ \\
\hline$x_{5}$ & & & & & 1,0933 & 0,6157 \\
\hline$x_{6}$ & & & & & & 1,0050 \\
\hline
\end{tabular}

Наибольший частный коэффициент корреляции (табл. 5) между третьим и пятым факторами (он равен 0,649 ), т. е. между общей площадью квартиры и площадью кухни существует очень тесная связь. Это говорит, что с увеличением площади кухни повышается и общая площадь квартиры. Самый низкий коэффициент между вторым и седьмым факторами (он равен 0,0096$)$, т. е. между количеством комнат и материалом дома, практически нет никакой зависимости.

3 способ. В данном способе используем критерий Фишера-Иейтса ${ }^{3}$ [2, с. 371]. Необходимо по заданному уровню значимости $\alpha=0,05$ и числу степеней свободы $d f=n-1-2=134$ выбирать $r_{\kappa p}$ из таблицы критерия Фишера-Иейтса. Для исследуемых данных, представленных в Интернет-проекте «Вся недвижимость Иркутска» ${ }^{4}$, критическое значение критерия составило $r_{\kappa p} \approx 0,18$. Сравнивая полученные результаты, приведенные в табл. 5 с критическим значением $\left|r_{i j}\right|>r_{\kappa p}$, получаем, что основную гипотезу $H_{0}$ следует отвергнуть для коэффициентов $r_{23 / . . .}$, $r_{24 / \ldots}, r_{34 / \ldots}, r_{25 / \ldots}, r_{35 / \ldots}, r_{45 / \ldots}, r_{17 / \ldots}$, а все остальные коэффициенты незначимы и соответствующими факторами можно пренебречь.

В результате анализа коэффициентов частной корреляции все три способа показали одинаковые результаты, что коэффициенты $r_{23 / . .,}, r_{24 / . .}, r_{34 / \ldots}, r_{25 / \ldots}, r_{35 / \ldots}$, $r_{45 / \ldots,}, r_{17 / \ldots .}$ значимы.

Следовательно, из проведенного анализа можно сделать вывод, что факторы: количество комнат квартиры, общая площадь, жилая площадь, площадь кухни и материал дома большим образом влияют на формирование цены квартиры.

Для количественных факторов были построены еще нелинейные модели [8, с. 75].

Степенная модель: $\hat{y}_{i}=22,53 \cdot\left(x_{1}\right)^{0,33} \cdot\left(x_{2}\right)^{0,14}$, с коэффициентом детерминации $R^{2}=0,65$. Невысокое значение коэффициента объясняется малостью факторов, которые рассматривались при построении модели. Частная эластичность по фактору - жилая площадь $E_{x 1}=0,33$ свидетельствует о том, что при изменении площади на $1 \%$ от своего среднего уровня, цена на однокомнатную квартиру увеличится на $0,33 \%$ от своего среднего уровня.

Экспоненциальная модель: $\widehat{y}_{i}=54,7 \cdot(e)^{0,01 x_{1}} \cdot(e)^{0,02 x_{2}}$, с коэффициентом детерминации $R^{2}=0,67$. Таким образом, $67 \%$ вариации жилой площади квартиры и кухни объясняет продажную стоимость рассматриваемых квартир.

В результате проведенного исследования можно сделать вывод, что на формирование цены однокомнатной квартиры в моногороде Шелехов наиболее существенным фактором является площадь рассматриваемой квартиры. Построенные модели верифицированы и их можно использовать для формирования цены на рынке жилья в моногородах, а также для выбора оптимальной стратегии в поведении налогообложения.

${ }^{3}$ URL: http://www.studfiles.ru/preview/1720882/.

${ }^{4}$ URL: http://www.dom-irk.ru.

\section{Baikal Research Journal}


Список использованной литературы

1. Путко Б. А. Эконометрика : учебник / Б. А. Путко, Н. Ш. Кремер ; под ред. Н. Ш. Кремера. - 3-е изд., перераб. и доп. - М. : Юнити-Дана, 2012. - 329 с.

2. Мхитарян В. С. Эконометрика : учебник / В. С. Мхитарян, М. Ю. Архипова, В. П. Сиротин [и др.]. - М. : Проспект, 2008. - 380 с.

3. Домбровский В. В. Эконометрика / В. В. Домбровский. - М. : Нов. учебник, 2004. - 342 с.

4. Дуброва Т. А. Статистические методы прогнозирования / Т. А. Дуброва. - М. : Юнити-Дана, 2003. - 206 с.

5. Колеников С. О. Прикладной эконометрический анализ в статистическом пакете Stata / С. О. Колеников. - М. : Рос. экон. шк., 2001. - 46 с.

6. Кремер Н. Ш. Эконометрика / Н. Ш. Кремер, Б. А. Путко. - М. : Юнити-Дана, 2002. -311 c.

7. Магнус Я. Р. Эконометрика. Начальный курс / Я. Р. Магнус, П. К. Катышев, А. А. Пересецкий. - 5-е изд., испр. - М. : Дело, 2000. -400 с.

8. Елисеева И. И. Эконометрика / И. И. Елисеева. - М. : Юрайт, 2012. - 450 с.

9. Овчаренко Е. К. Финансово-экономические расчеты в Excel / Е. К. Овчаренко, О. П. Ильина, Е. В. Балыбердин. - М. : Филинъ, 1998. - 328 с.

10. Айвазян С. А. Основы эконометрики / С. А. Айвазян, В. С. Мхитарян. - М. : Юнити-Дана, 2001. - 432 с.

\section{References}

1. Putko B. A., Kremer N. Sh. Ekonometrika [Economentrics]. $3^{\text {rd }}$ ed. Moscow, Yuniti-Dana Publ., 2012. 329 p.

2. Mkhitaryan V. S., Arkhipova M. Yu., Sirotin V. P. et al. Ekonometrika [Econometrics]. Moscow, Prospekt Publ., 2008. 380 p.

3. Dombrovsky V. V. Ekonometrika [Econometrics]. Moscow, Novyi uchebnik Publ., 2004. 342 p.

4. Dubrova T. A. Statisticheskie metody prognozirovaniya [Statistical Methods of Forecasting]. Moscow, Yuniti-Dana Publ., 2003. 206 p.

5. Kolenikov S. O. Prikladnoi ekonometricheskii analiz $v$ statisticheskom pakete Stata [Applied econometric analysis in the Stata statistic package]. Moscow, Rossiiskaya ekonomicheskaya shkola Publ., 2001. 46 p.

6. Kremer N. Sh., Putko B. A. Ekonometrika [Econometrics]. Moscow, Yuniti-Dana Publ., 2002. $311 \mathrm{p}$.

7. Magnus Ya. R., Katyshev P. K., Peresetsky A. A. Ekonometrika. Nachal'nyi kurs [Econometrics. A Beginning Course]. 5 ${ }^{\text {th }}$ ed. Moscow, Delo Publ., 2000. 400 p.

8. Eliseyeva I. I. Ekonometrika [Econometrics]. Moscow, Yurait Publ., 2012. 450 p.

9. Ovcharenko E. K., Ilyina O. P., Balyberdin E. V. Finansovo-ekonomicheskie raschety $v$ Excel [Financial and economic calculations in Exel]. Moscow, Filin” Publ., 1998. 328 p.

10. Aivazyan S. A., Mkhitaryan V. S. Osnovy econometriki [Basics of Econometrics]. Moscow, Yuniti-Dana Publ., 2001. 432 p.

\section{Информация об авторе}

Малонова Наталья Вячеславовна - кандидат физико-математических наук, доцент, кафедра математики и эконометрики, Байкальский государственный университет, 664003, г. Иркутск, ул. Ленина, 11, e-mail: naamm@mail.ru.

\section{Author}

Natalya V. Mamonova - PhD in Physics and Mathematics, Associate Professor, Chair of Mathematics and Econometrics, Baikal State University, 11 Lenin St., 664003, Irkutsk, Russian Federation; e-mail: naamm@mail.ru.

\section{Библиографическое описание статьи}

Мамонова Н. В. Исследование рынка недвижимости в городе Шелехове / Н. В. Мамонова // Baikal Research Journal. — 2017. — T. 8, № 1. - DOI: 10.17150/2411-6262.2017.8(1).14.

\section{Reference to article}

Mamonova N. V. Investigation of real estate market in the Shelekhov town. Baikal Research Journal, 2017, vol. 8, no. 1. DOI: 10.17150/2411-6262.2017.8(1).14. (In Russian).

\section{Baikal Research Journal}

